\title{
Research Article \\ Stability for Linear Volterra Difference Equations in Banach Spaces
}

\author{
Rigoberto Medina \\ Departamento de Ciencias Exactas, Universidad de Los Lagos, Casilla 933, Osorno, Chile \\ Correspondence should be addressed to Rigoberto Medina; rmedina@ulagos.cl
}

Received 27 October 2017; Revised 23 December 2017; Accepted 16 January 2018; Published 1 March 2018

Academic Editor: Changbum Chun

Copyright (c) 2018 Rigoberto Medina. This is an open access article distributed under the Creative Commons Attribution License, which permits unrestricted use, distribution, and reproduction in any medium, provided the original work is properly cited.

This paper is devoted to studying the existence and stability of implicit Volterra difference equations in Banach spaces. The proofs of our results are carried out by using an appropriate extension of the freezing method to Volterra difference equations in Banach spaces. Besides, sharp explicit stability conditions are derived.

\section{Introduction}

In this paper, we study the existence and stability of solutions for a class of abstract functional difference equations described in the form

$$
x(k)=\sum_{j=0}^{k} K(k, k-j) x(j)+f(k), \quad k \geq 1
$$

in a Banach space $(X,\|\cdot\|), f \in l_{\infty}\left(Z^{+}, X\right)$, the space of bounded sequences equipped with the norm $\|\cdot\|$ on $l_{\infty}$, and $K(k, j)$ is a function defined on $0 \leq j \leq k<\infty$, whose values are bounded operators in $X$. In addition, for any fixed integer $\tau \geq 0, K(\tau, \cdot)$ is summable and bounded on $Z^{+}$, the set of nonnegative integers.

A solution of (1) is a sequence defined on $Z^{+}$and satisfying (1) for all finite $k>0$. The study of existence and stability of solutions for implicit Volterra difference equations of nonconvolution type, defined in abstract spaces, is a complicated problem. However, with appropriate conditions on $f(\cdot)$ and $K(k, \cdot)$, one can use the freezing method for abstract Volterra difference equations, so the difficulty is overcome.

Existence and uniqueness problems for the Volterra difference equations were discussed by some authors (e.g., see $[1,2])$. Existence and stability of Volterra difference equations have been studied by many authors (Federson et al. [3], Murakami and Nagabuchi [4], Györi and Horvath [5],
Mingarelli [1], Gonzalez et al. [6], Kolmanovskii et al. [7], and Song and Baker [8]).

The main technique in the theory of stability and boundedness of Volterra difference equations is the direct Lyapunov method and its variants. In contrast, many alternative methods to Lyapunov's function have been successfully applied to the stability analysis of Volterra difference equations; for example, in Federson et al. [3], the Kurzweil-Henstok integral formalism is applied to establish the existence of solutions to integral equations of Volterra type. In Murakami and Nagabuchi [4], sufficient stability properties and the asymptotic almost periodicity for linear Volterra difference equations in Banach spaces are derived. Gonzalez et al. [6] considered an implicit Volterra difference equation in a Hilbert space and obtained sufficient conditions so that the solutions exist and have a bounded behavior. The coefficients of the considered equations are sequences of real numbers. In Mingarelli [1], Volterra-Stieltjes integral equations are studied, which can be considered as generalized Volterra difference equations. In Banás and Sadarangani [2], a class of operator-integral equations of Volterra-Stieltjes type which create a generalization of numerous integral equations appearing in mathematical literature is studied. In Györi and Horvath [5], sufficient conditions are presented under which the solutions to a linear nonconvolution Volterra difference equation converge to limits, which are given by a limit formula. In Kolmanovskii et al. [7], stability and boundedness problems of some classes of scalar Volterra 
nonlinear difference equations are investigated. Their stability conditions are formulated in terms of the characteristic equations. In Song and Baker [8], the fixed point theory is used to establish sufficient conditions to ensure the stability of the zero solution of an implicit nonlinear Volterra difference equation. However, in the above-mentioned articles, Volterra equations with convolution kernels are mainly considered.

In this paper, formulating the Volterra discrete equations in the phase space $l_{p}\left(Z^{+}, X\right)$, where $X$ is an appropriate Hilbert space, and assuming that the kernel operator is completely continuous, we obtain sufficient conditions for the existence and uniqueness problem. The suggested approach is based on the "freezing" method to abstract difference equations (Medina and Gil' [9]), as well as on the concept of analytical pencils (analytic operator-valued functions of a complex argument). See, for example, [10-13]. In Medina [14], a class of nonlinear discrete-time Volterra equations in Banach spaces is considered. Using a linearization method, sufficient conditions of existence and boundedness are established. In fact, assuming that the kernels are Causal Operators, the existence and boundedness of solutions are derived. Consequently, the methodology and the corresponding results obtained in [14] are absolutely different compared with the results of this article. form

Consider an $X$-valued Volterra-Stieltjes equation of the

$$
x(t)=\int_{0}^{t} K(t, s, x(s)) d \mu(s)+f(t), \quad t \geq 0,
$$

where $K:[0, \infty) \times[0, \infty) \times X \rightarrow X$ and $f: L_{\mu, \infty} \rightarrow L_{\mu, \infty}$. A solution of this equation is a function $x \in L_{\mu, \infty}$, which is locally $\mu$-integrable in the Riemann-Stieltjes sense.

If $\mu(t)=k-1, k-1<t \leq k$, for $k=1,2, \ldots$, then we can restrict our attention to functions $x \in L_{\mu, \infty}$ which are piecewise constants with $x(t)=x_{k}$ for $k-1<$ $t \leq k, k=1,2, \ldots$. We can identify such a function $x$ with a sequence $\left(x_{1}, x_{2}, \ldots\right)$ in the space $\ell_{\infty}$. In this case, the Volterra-Stieltjes original equation is equivalent to a Volterra difference equation

$$
x(j)=\sum_{k=1}^{j} K(j, k, x(k))+f(j), \quad j=2,3, \ldots
$$

Consequently, $[1,2,15,16]$ are highly appropriate to our research.

Remark 1. We want to point out that the freezing method was introduced by V. M. Alekseev for linear ordinary differential equations (see Bylov et al. [17]) and extended to difference systems by Gil' and Medina [18].

Our aim in this paper is to make new contributions to the development of the theory of existence and qualitative properties of solutions for the nonconvolution Volterra difference equations described by Volterra operators in Banach spaces.

The remainder of this article is organized as follows: In Section 2, we establish a preliminary result to a class of convolution Volterra difference equations which will be fundamental to formulating the corresponding nonconvolution problem in Banach spaces. In Section 3, sufficient conditions on the existence and stability of solutions of nonconvolution Volterra difference equations are established. In Section 4, we illustrate the main result studying an interesting problem. Finally, Section 5 is devoted to the discussion of our results.

\section{Preliminary Results}

To prove our main results, it is convenient to establish some known qualitative results for a class of convolution Volterra difference equations (see [7, 8, 11, 19-25]).

Let $K(i), i=0,1,2, \ldots$, be bounded linear operators in a Banach space $X$ with norm $\|\cdot\|$.

Consider the convolution Volterra difference equation

$$
x(k)=\sum_{j=1}^{k} K(k-j) x(j)+h(k) ; \quad k=1,2, \ldots
$$

where $K(0)=0, h=\{h(i)\}_{i=1}^{\infty}$, and $h(i) \in X$ is a given sequence.

Assume that $\varlimsup_{k \rightarrow \infty} \sqrt[k]{\|K(k)\|}<\infty$ and $\varlimsup_{\lim _{k \rightarrow \infty}}^{\sqrt[k]{\|h(k)\|}}<$ $\infty$.

To solve (4), put

$$
\begin{aligned}
T(z) & =\sum_{j=1}^{\infty} K(j) z^{j}, \\
f(z) & =\sum_{j=1}^{\infty} h(j) z^{j}, \quad(z \in \mathbb{C}) .
\end{aligned}
$$

Consider the equation

$$
y(z)=T(z) y(z)+f(z) .
$$

In a neighborhood $\omega$ of zero, let $I-T(z)$ be boundedly invertible. Then

$$
y(z)=(I-T(z))^{-1} f(z), \quad(z \in \omega)
$$

Hence it follows that $y(z)$ is infinitely many times differentiable at zero.

Differentiating (6) $j$ times, we get

$$
y^{(j)}(z)=\sum_{i=0}^{j} C_{j}^{i} T^{(j-i)}(z) y^{(i)}(z)+f^{(j)}(z) .
$$

Since $K(i)=T^{(i)}(0) / i$ !, substituting $z=0$ into the later equality, we obtain the following relations:

$$
b(j)=\sum_{i=0}^{j} K(j-i) b(i)+h(j)
$$

$$
(K(0)=0 ; j=1,2, \ldots)
$$


where $b(j)=y^{(j)}(0) / j$ !. Therefore, we arrive at (7). Hence, the sequence $x(k)=b(k)$ is a solution to (7). According to (7), we obtain

$$
\begin{aligned}
x(j) & =\left.\frac{1}{j !} \frac{d^{j} y(z)}{d z^{j}}\right|_{z=0} \\
& =\left.\frac{1}{j !} \frac{d^{j}}{d z^{j}}(1-T(z))^{-1} f(z)\right|_{z=0} .
\end{aligned}
$$

Thanks to the Cauchy formula

$$
\begin{aligned}
x(j)=\frac{1}{2 \pi i} \int_{\gamma} \frac{1}{z^{j+1}}(1-T(z))^{-1} f(z) d z, & \\
& (j=1,2, \ldots),
\end{aligned}
$$

where $\gamma$ is a smooth contour surrounding zero, provided that $I-T(z)$ is boundedly invertible and $f$ is regular inside $\gamma$ and on $\gamma$. Thus, the next result can be established.

Theorem 2 (see $[19,25]$ ). Inside $\gamma$ and on $\gamma$, let $I-T(z)$ be boundedly invertible and $f$ be regular. Then a solution of (4) is given by formula (11).

Remarks 3. Theorem 2 will play a fundamental role to establish the existence and stability of the solution of nonconvolution equations of kind (1). In doing so, we will use the freezing method.

Definition 4 (see [7, 8, 22-24]). We will say that (1) is stable if, for any $f \in l_{\infty}\left(Z^{+}, X\right)$, a solution $x$ of (1) satisfies the inequality

$$
\|x\|_{l_{\infty}} \leq c_{0}\|f\|_{l_{\infty}}
$$

where the constant $c_{0}$ does not depend on $f$.

Let $H$ be a separable Hilbert space and $A$ a linear compact operator in $H$. If $\left\{e_{k}\right\}_{k=1}^{\infty}$ is an orthogonal basis in $H$ and the series $\sum_{k=1}^{\infty}\left(A e_{k}, e_{k}\right)$ converges, then the sum of the series is called the trace of the operator $A$ and is denoted by

$$
\operatorname{Trace}(A)=\operatorname{Tr}(A)=\sum_{k=1}^{\infty}\left(A e_{k}, e_{k}\right) .
$$

Definition 5 (see $[20,26]$ ). An operator $A$ satisfying the relation $\operatorname{Tr}\left(A^{*} A\right)<\infty$ is said to be a Hilbert-Schmidt operator, where $A^{*}$ is the adjoint operator of $A$.

The norm

$$
N_{2}(A)=N(A)=\sqrt{\operatorname{Tr}\left(A^{*} A\right)}
$$

is called the Hilbert-Schmidt norm of A.

Definition 6 (see $[20,26])$. A bounded linear operator $A$ is said to be quasi-Hermitian if its imaginary component

$$
A_{I}=\frac{A-A^{*}}{2 i}
$$

is a Hilbert-Schmidt operator, where $A^{*}$ is the adjoint operator of $A$.
Theorem 7 (see $[17,19,26])$. Let $V$ be a Hilbert-Schmidt completely continuous quasinilpotent (Volterra) operator acting in a separable Hilbert space $H$. Then the inequality

$$
\left\|V^{k}\right\| \leq \frac{N_{p}^{k}(V)}{\sqrt{k !}}, \text { for any natural } k
$$

is true.

\section{Main Results}

Now, we are in a position to establish sufficient conditions on the existence and stability of solutions of (1).

Assume that, for any fixed integer $\tau \geq 0, K(\tau, \cdot)$ is $l_{1}$ summable and bounded on $Z^{+}$. In addition, assume that there exists a nonnegative constant $q$ such that

$$
\begin{array}{r}
\sum_{j=0}^{k}\|K(k, j)-K(\tau, j)\| \leq q|k-\tau|, \\
\quad(q=\text { const.; } k, \tau \geq 0) .
\end{array}
$$

Under (17), the function $K(\tau, j)$, for a fixed integer $\tau$, admits the $Z$-transform $\widetilde{K}_{\tau}(z)=\sum_{j=0}^{\infty} z^{-j} K(\tau, j),|z| \geq \rho$, where $\rho$ is the radius of convergence of $\widetilde{K}_{\tau}(z)$. Besides, it is assumed that the operator $W_{\tau}(z)=1-\widetilde{K}_{\tau}(z)$ is boundedly invertible for all $z$ in a neighborhood $\omega$ of zero.

Introduce the Green function

$$
G_{\tau}(k)=\frac{1}{2 \pi} \int_{0}^{2 \pi} e^{-i y k} W_{\tau}^{-1}\left(e^{i y}\right) d y .
$$

Theorem 8. Under assumption (17), let

$$
q \sum_{k=0}^{\infty} k \sup _{\tau \geq 0}\left\|G_{\tau}(k)\right\|<1 .
$$

Then (1) is stable. Moreover, constant $c_{0}$ in (12) is explicitly pointed below.

Proof. Consider the convolution equation

$$
x(k)=\sum_{j=0}^{k} K(\tau, k-j) x(j)+f(k), \quad k \geq 1,
$$

with a fixed integer $\tau \geq 0$.

The solution of (20) may be written as

$$
x(k)=\sum_{j=1}^{k} G_{\tau}(k-j) f(j),
$$

whereby the discrete Green's function $G(k, j)$ is defined.

Now, rewrite (20) in the form

$$
x(k)-\sum_{j=1}^{k} K(\tau, k-j) x(j)=f_{0}(k, \tau)+f(k),
$$

$$
(k \geq 0) \text {, }
$$


where

$$
f_{0}(k, \tau)=\sum_{j=1}^{k}[K(k, k-j)-K(\tau, k-j)] x(j) .
$$

Hence, according to (21),

$$
\begin{aligned}
x(k) & =\sum_{j=1}^{k} G_{\tau}(k-j)\left[f(j)+f_{0}(j, k)\right], \\
& =F(k)+\sum_{j=1}^{k} G_{\tau}(k-j) f_{0}(j, k),
\end{aligned}
$$

where $F(k)=\sum_{j=1}^{k} G_{\tau}(k-j) f(j)$.

Denote

$$
M(k)=\sup _{\tau \geq 0}\left\|G_{\tau}(k)\right\| .
$$

Hence, we get

$$
\|F\|_{l_{\infty}} \leq\|f\|_{l_{\infty}} \sup _{k}\left(\sum_{j=1}^{k} M(k-j)\right) \leq\|M\|_{l_{\infty}}\|f\|_{l_{\infty}} .
$$

Due to $(22)$,

$$
\begin{aligned}
\left\|f_{0}(k, \tau)\right\| & \leq \sum_{j=1}^{k}\|K(k, k-j)-K(\tau, k-j)\|\|x(j)\| \\
& \leq q\|x\|_{l_{\infty}}|k-\tau| .
\end{aligned}
$$

By (24), we have

$$
\|x(k)\| \leq\|M\|_{l_{\infty}}\|f\|_{l_{\infty}}+q\|x\|_{l_{\infty}} \sum_{j=1}^{k} M(k-j)|j-\tau| .
$$

Take $k=\tau$; then

$$
\begin{aligned}
\|x(\tau)\| \leq & \|M\|_{l_{\infty}}\|f\|_{l_{\infty}} \\
& +q \sup _{1 \leq j \leq \tau}\|x(j)\| \sum_{j=1}^{\tau}(\tau-j) M(\tau-j) .
\end{aligned}
$$

Hence

$$
\|x(\tau)\| \leq\|M\|_{l_{\infty}}\|f\|_{l_{\infty}}+q \sup _{1 \leq j \leq \tau}\|x(j)\| \sum_{j=1}^{\infty} j M(j) .
$$

Therefore, for any $k_{0}>0$,

$$
\sup _{\tau \leq k_{0}}\|x(\tau)\| \leq\|M\|_{l_{\infty}}\|f\|_{l_{\infty}}+\lambda \sup _{\tau \leq k_{0}}\|x(\tau)\|,
$$

where $\lambda=q \sum_{j=1}^{\infty} j M(j)$.

On the other hand, condition $q \sum_{j=1}^{\infty} j M(j)<1$ implies

$$
\sup _{1 \leq k \leq k_{0}}\|x(k)\| \leq \frac{\|M\|_{l_{\infty}}\|f\|_{l_{\infty}}}{1-\lambda} \text {. }
$$

Since the right hand side does not depend on $k_{0}$, condition (12) follows with $c_{0}=\|M\|_{l_{\infty}} /(1-\lambda)$.

The existence of solutions is due to the convergence of the Neumann series

$$
x=\sum_{j=0}^{\infty} V^{j} f
$$

where

$$
(V x)(k)=\sum_{j=1}^{k} K(k, j) x(j)
$$

provided that $\sum_{k=1}^{\infty}\|K(k, \tau)\|<\infty$ for any fixed integer $\tau \geq 0$. In fact, (1) is rewritten in the operator form

$$
x=V x+f
$$

Hence

$$
x=(I-V)^{-1} f=\sum_{j=0}^{\infty} V^{j} f .
$$

This yields

$$
\|x\|_{l_{\infty}} \leq \sum_{j=0}^{\infty}\left\|V^{j}\right\|_{l_{\infty}}\|f\|_{l_{\infty}} .
$$

Since $V$ is a quasinilpotent Hilbert-Schmidt operator, it follows by $[19,25]$ that

$$
\left\|V^{j}\right\|_{l_{\infty}} \leq \frac{N_{\infty}^{p}(V)}{\sqrt[p]{k !}}<\infty .
$$

Consequently, the Neumann series $\sum_{j=0}^{\infty} V^{j} f$ is convergent.

Remark 9. The stability theory of Volterra difference equations has been considered, for example, by Song and Baker [8], Mingarelli [1], and Gonzalez et al. [6]. However, the "freezing" method has not been used previously to study qualitative properties of Volterra difference systems in Banach spaces. Consequently, the theoretical contributions of this paper are significantly new.

\section{Example}

To illustrate the main result, consider in $X$ the equation

$$
x(k)-A(k) \sum_{j=0}^{k} e^{-(k-j) h} x(j)=f(k)
$$

$$
h=\text { const. }>0 \text {, }
$$

where $A(k)$ is a variable bounded operator in $X$ satisfying

$$
\|A(k)-A(\tau)\| \leq q_{0}|k-\tau| ; \quad(k, \tau \geq 0) .
$$


Take $K(k, j)=A(k) e^{-j h}$. Then, by (41),

$$
\begin{aligned}
& \sum_{j=0}^{k}\|K(k, j)-K(\tau, j)\| \leq\|A(k)-A(\tau)\| \sum_{j=0}^{k} e^{-j h} \\
& \quad \leq \frac{q_{0}}{e^{h}}|k-\tau| .
\end{aligned}
$$

We also have

$$
\begin{aligned}
\widetilde{K}_{\tau}(z) & =\sum_{j=0}^{\infty} \frac{K(\tau, j)}{z^{j}}=A(\tau) \sum_{j=0}^{\infty} \frac{\left(e^{-h}\right)^{j}}{z^{j}} \\
& =A(\tau) \frac{z}{z-e^{-h}}, \quad|z|>\frac{1}{e^{h}} .
\end{aligned}
$$

This relation yields

$$
W_{\tau}(z)=I-\frac{A(\tau) z}{z-e^{-h}} .
$$

On the other hand,

$$
\begin{aligned}
k G_{\tau}(k) & =k\left(\frac{1}{2 \pi i} \int_{\gamma} z^{k-1} W_{\tau}^{-1}(z) d z\right) \\
& =\frac{1}{2 \pi i} \int_{\gamma} z^{k-1} T_{\tau}(z) d z,
\end{aligned}
$$

where

$$
T_{\tau}(z)=-\frac{d}{d z}\left(W_{\tau}^{-1}(z)\right) .
$$

If $|z|<b<1$ and letting $T_{\tau}(z)$ be regular and

$$
\lambda_{b}=\sup _{\tau \geq 0} \frac{1}{2 \pi} \int_{0}^{2 \pi}\left\|T_{\tau}\left(e^{i y-b}\right)\right\| d y<\infty,
$$

then

$$
\left\|k G_{\tau}(k)\right\| \leq b^{k-1} \frac{1}{2 \pi} \int_{0}^{2 \pi}\left\|T_{\tau}\left(e^{i y-b}\right)\right\| d y \leq b^{k-1} \lambda_{b} .
$$

Consequently,

$$
\sum_{k=0}^{\infty} k \sup _{\tau \geq 0}\left\|G_{\tau}(k)\right\| \leq \frac{\lambda_{b}}{b(1-b)} .
$$

Theorem 10. Under conditions (42), for a positive $b$ and all $z$, with $|z|<b<1$, let $T_{\tau}(z)$ be regular and $\lambda_{b} / b(1-b)<1$. Then (40) is stable.

For example, if $A(t)=\sin (t) B$, where $B$ is a constant operator, then condition (41) holds with

$$
q(t)=2\|B\| \sin \left(\frac{t}{2}\right)
$$

since

$$
\sin \alpha-\sin \beta=2 \sin \left(\frac{\alpha-\beta}{2}\right) \cos \left(\frac{\alpha+\beta}{2}\right),
$$

Taking $K(k, j)=\sin (t) B e^{-j h}$. Then, by (41),

$$
\begin{aligned}
& \sum_{j=0}^{k}\|K(k, j)-K(\tau, j)\| \leq\|A(k)-A(\tau)\| \sum_{j=0}^{k} e^{-j h} \\
& \quad \leq \frac{2\|B\|}{e^{h}}|k-\tau| .
\end{aligned}
$$

Thus, every statement of Theorem 10 can be easily verified.

\section{Concluding Remarks}

The stability problem for Volterra difference equations of nonconvolution type in an infinite dimensional Hilbert space is more complicated than that for equations in $R^{n}$ (a finite dimensional Euclidean space). However, with appropriate conditions on $f(\cdot)$ and $K(j, \cdot)$, one can use the freezing method for abstract difference equations, so the difficulty is overcome. In fact, considering the time $j$ as a parameter, we obtain an infinite family of convolution Volterra difference equations. Thus, using the freezing method, we deduce the qualitative properties corresponding to the nonconvolution Volterra difference equations and to the convolution original equation. On the other hand, the study of existence of solutions of this kind of implicit Volterra difference equations is a complicated problem. Our proof of the existence of solutions is carried out using the convergence of Neumann series of quasinilpotent Hilbert-Schmidt operators [27].

\section{Conflicts of Interest}

The author declares that there are no conflicts of interest regarding the publication of this paper.

\section{Acknowledgments}

This research was supported by Dirección de Investigación under Grant NU06/16.

\section{References}

[1] A. B. Mingarelli, Volterra-Stieltjes Integral Equations and Generalized Ordinary Differential Expressions, vol. 989 of Lecture Notes in Mthematics, Springer-Verlag, Berlin, Germany, 1983.

[2] J. Banás and K. Sadarangani, "Solvability of Volterra-Stieltjes operator-integral equations and their applications," Computers \& Mathematics with Applications. An International Journal, vol. 41, no. 12, pp. 1535-1544, 2001.

[3] M. Federson, R. Bianconi, and L. Barbanti, "Linear Volterra integral equations," Acta Mathematicae Applicatae Sinica, vol. 18, no. 4, pp. 553-560, 2002.

[4] S. Murakami and Y. Nagabuchi, "Stability properties and asymptotic almost periodicity for linear Volterra difference equations in a Banach space," Japanese Journal of Mathematics, vol. 31, no. 2, pp. 193-223, 2005.

[5] I. Györi and L. Horvath, "Asymptotic representation of the solutions of linear volterra difference equations," Advances in Difference Equations, vol. 2008, Article ID 932831, 2008. 
[6] C. Gonzalez, A. Jimenez-Melado, and M. Lorente, "Existence and estimate of solutions of some nonlinear Volterra difference equations in Hilbert spaces," Journal of Mathematical Analysis and Applications, vol. 305, no. 1, pp. 63-71, 2005.

[7] V. B. Kolmanovskii, A. D. Myshkis, and J.-P. Richard, "Estimate of solutions for some Volterra difference equations," Nonlinear Analysis: Theory, Methods \& Applications. An International Multidisciplinary Journal, vol. 40, no. 1-8, pp. 345-363, 2000.

[8] Y. Song and C. T. Baker, "Perturbation theory for discrete Volterra equations," Journal of Difference Equations and Applications, vol. 9, no. 10, pp. 969-987, 2003.

[9] R. Medina and M. I. Gil', “The freezing method for abstract nonlinear difference equations," Journal of Mathematical Analysis and Applications, vol. 330, no. 1, pp. 195-206, 2007.

[10] F. V. Atkinson, H. Langer, and R. Mennicken, "Sturm-Liouville problems with coefficients which depend analytically on the eigenvalue parameter," Acta Universitatis Szegediensis: Acta Scientiarum Mathematicarum, vol. 57, no. 1-4, pp. 25-44, 1993.

[11] H. O. Fattorini, Second Order Linear Differenttial Equations in Banach spaces, Elsevier, Amsterdam, Netherlands, 1996.

[12] A. E. Lifschitz, Magneto-hydrodynamics and Spectral Theory, Kluwer Academic Publishers, Dordrecht, Netherlands, 1989.

[13] J. Wu, Theory and Applications of Partial Functional-Differential Equations, Springer, New York, NY, USA, 1996.

[14] R. Medina, "Existence and boundedness of solutions for nonlinear volterra difference equations in banach spaces," Abstract and Applied Analysis, vol. 2016, Article ID 1319049, 2016.

[15] P. H. A. Ngoc, "Stability of linear Volterra-Stieltjes differential equations," SIAM Journal on Control and Optimization, vol. 49, no. 1, pp. 205-226, 2011.

[16] J. Banás and D. ORegan, "Volterra-Stieltjes integral operators," Mathematical and Computer Modelling, vol. 41, no. 2-3, pp. 335344, 2005.

[17] B. F. Bylov, B. M. Grobman, V. V. Nemyckii, and R. E. Vinograd, The Theory of Lyapunov Exponents, Nauka, Moscow, Russia, 1966.

[18] M. I. Gil' and R. Medina, “The freezing method for linear difference equations," Journal of Difference Equations and Applications, vol. 8, no. 5, pp. 485-494, 2002.

[19] M. I. Gil', Norm Estimations for Operator-Valued Functions and Applications, vol. 192 of Monographs and Textbooks in Pure and Applied Mathematics, Marcel Dekker, New York, NY, USA, 1995.

[20] C. Corduneanu, Functional Equations with Causal Operators, vol. 16, Taylor and Francis, London, UK, 2002.

[21] V. B. Kolmanovskii and A. I. Matasov, "An approximate method for solving optimal control problem in hereditary systems," Doklady Math, vol. 55, no. 3, pp. 475-478, 1997.

[22] N. Levinson, "A nonlinear Volterra equation arising in the theory of superfluidity," Journal of Mathematical Analysis and Applications, vol. 1, pp. 1-11, 1960.

[23] Y. Nagabuchi, "Decomposition of phase space for linear Volterra difference equations in a Banach space," Funkcialaj Ekvacioj. Serio Internacia, vol. 49, no. 2, pp. 269-290, 2006.

[24] P. Rejto and M. Taboada, "Unique solvability of nonlinear Volterra equations in weighted spaces," Journal of Mathematical Analysis and Applications, vol. 167, no. 2, pp. 368-381, 1992.

[25] H. König, Eigenvalue Distribution of Compact Operators, Birkhäuser Verlag, Basel, Switzerland, 1986.

[26] N. Dunford and J. T. Schwarz, Linear Operators, Part I, Wiley Interscience Publishers, New York, NY, USA, 1966.
[27] N. Suzuki, "On the convergence of Neumann series in Banach space," Mathematische Annalen, vol. 220, no. 2, pp. 143-146, 1976. 


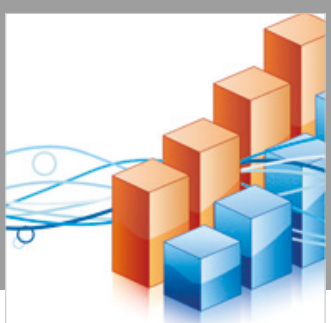

Advances in

Operations Research

\section{-n-m}
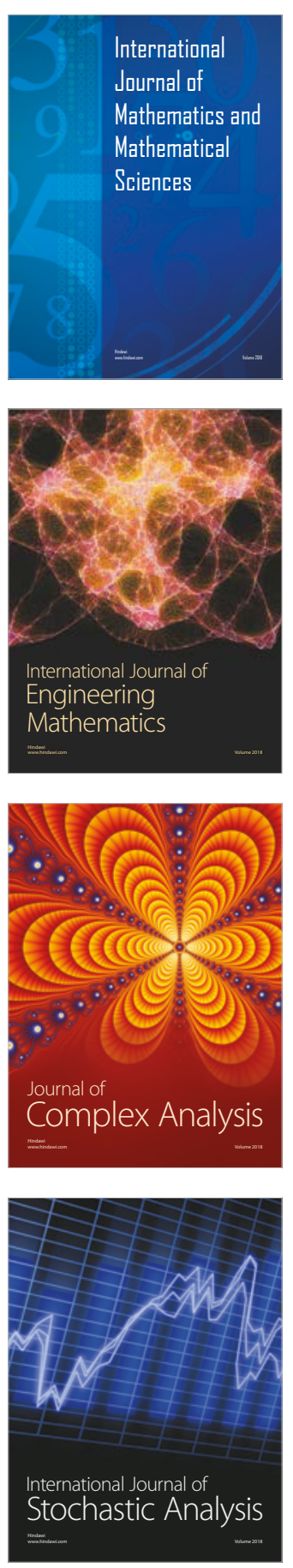
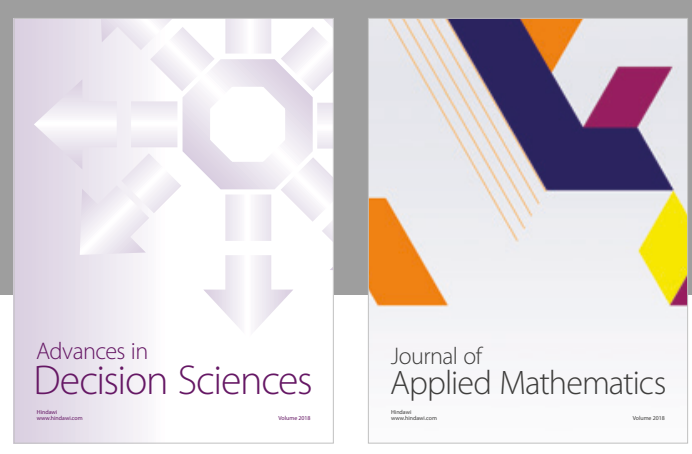

Journal of

Applied Mathematics
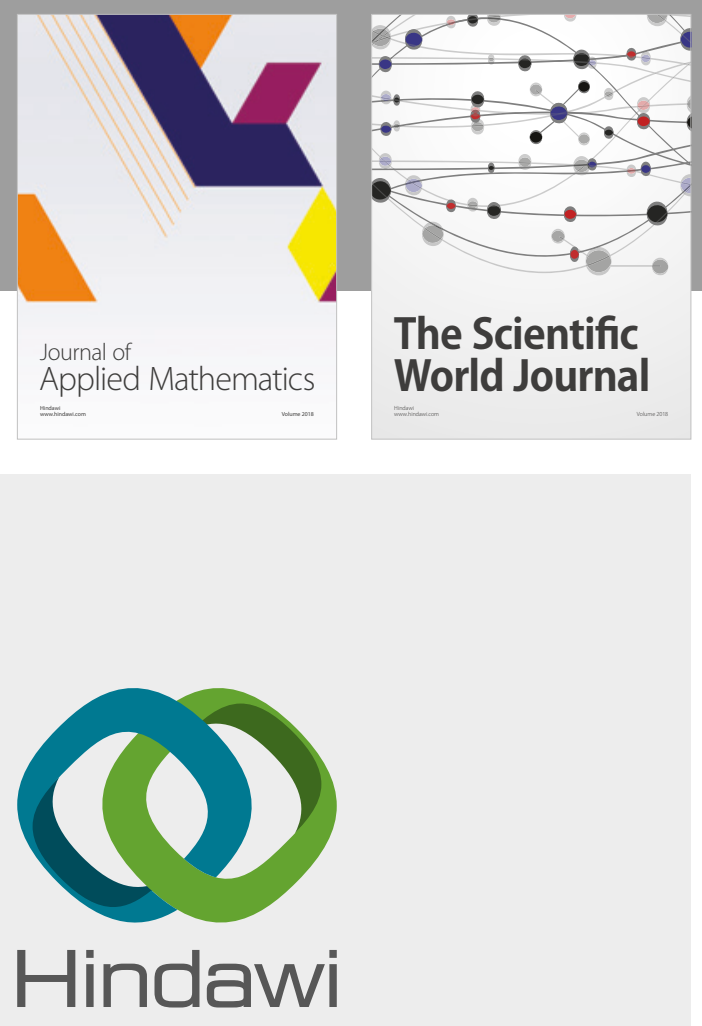

Submit your manuscripts at

www.hindawi.com

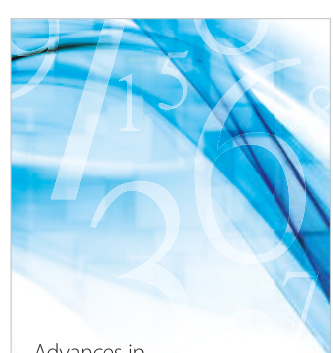

Advances in
Numerical Analysis
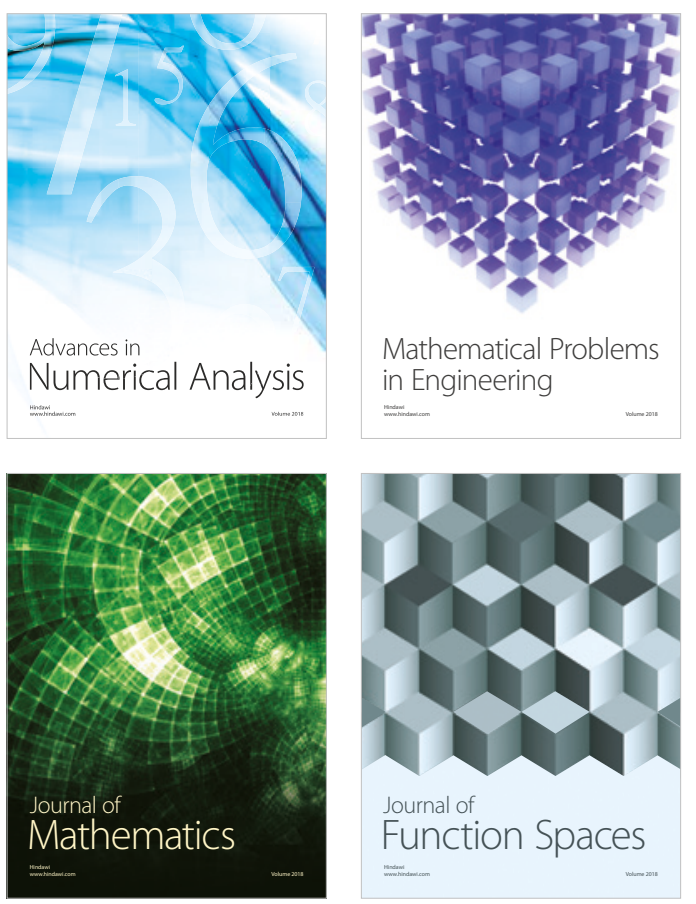

Mathematical Problems in Engineering

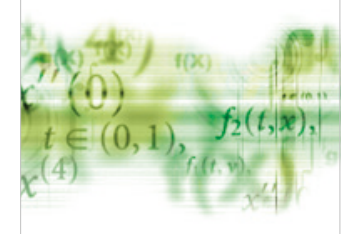

International Journal of

Differential Equations

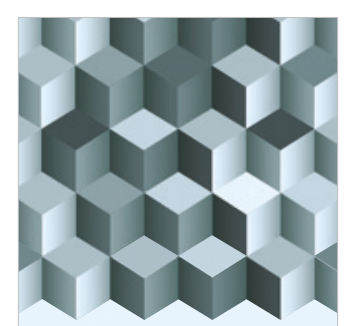

Journal of

Function Spaces

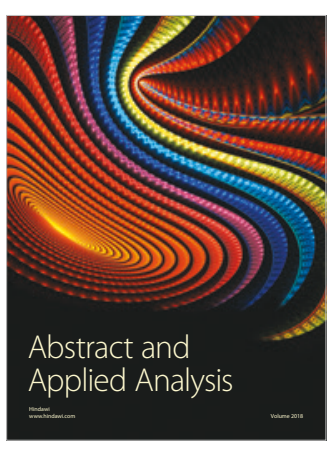

The Scientific

World Journal

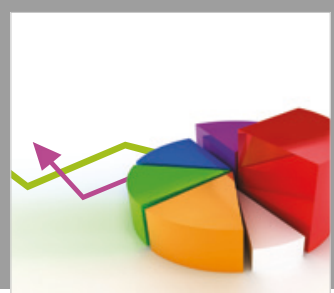

Journal of

Probability and Statistics
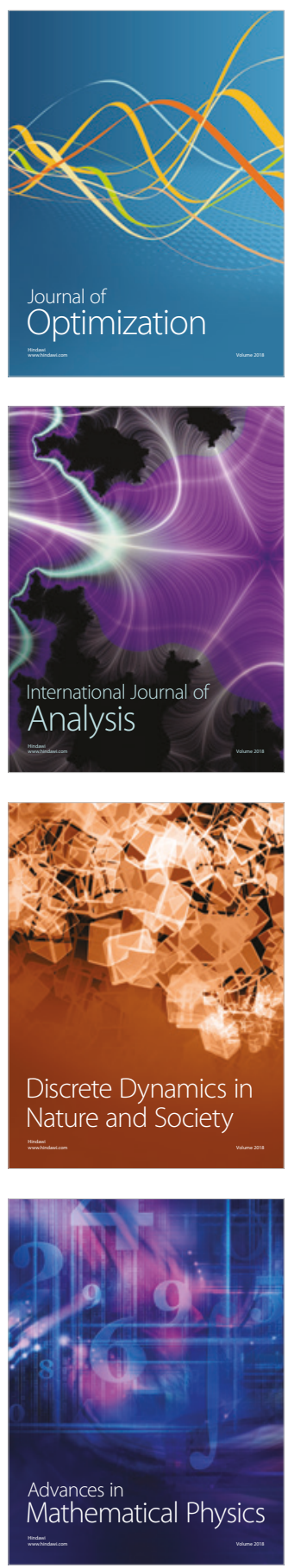